\title{
Estudio sobre la identidad magallánica antártica: cuando se idealiza lo desconocido ${ }^{1}$
}

\author{
Study on antarctic magellan identity: \\ when the unknown is idealized \\ Sebastián I. Legue G., Eric J. Pacheco A., \\ Víctor A. Ulloa S. \& Claudia A. Estrada G. \\ "Centro de Estudios en Psicología Social, Departamento de Psicología, Universidad de Magallanes \\ $\triangle$ claudia.estrada@umag.cl
}

\begin{abstract}
RESUMEN
El estudio se ocupa de la relación entre el conocimiento de un territorio al que se pertenece desde una perspectiva sociopolítica, la identidad asociada a los aspectos ambientales (Identidad Regional Ecológica), y variables socio-identitarias tales como el regionalismo y el esencialismo. Un total de 300 voluntarios habitantes de la Patagonia Austral participaron respondiendo instrumentos sobre conocimiento general respecto de la Antártica, identidad regional ecológica, regionalismo y esencialismo. Los resultados señalan que aunque la identificación de lo antártico como parte de la propia identidad social resultó vinculada al conocimiento sobre este territorio, las variables socio-identitarias mostraron vinculaciones más fuertes. Se discuten los resultados en torno a la importancia relativa de los factores emocionales y cognitivos en la construcción de la identidad social vinculada con el territorio.
\end{abstract}

PALABRAS CLAVE: identidad social, identidad regional, Antártica, nacionalismo, esencialismo, creencias.

\begin{abstract}
The study deals with the relationship among the knowledge about a territory that someone belongs to, from a sociopolitical perspective, the identity associated with the environmental aspects (Ecological regional identity), and socio-identity believe such as the regionalism and the essentialism. A total of 300 volunteers, who live in Austral Patagonia, participated answering instruments about general knowledge related to the Antarctica,

1 Investigación apoyada por el Convenio de Desempeño GAIA-Antártica, MINEDUC.
\end{abstract}


ecological regional identity, regionalism, and essentialism. The results show that although the identification of the Antarctic as part of the own social identity resulted to be linked to the knowledge about the territory, the socio-identity variables show to be stronger. The results are discussed around the relative importance of the emotional and cognitive factors in the construction of the social identity linked with the territory.

KEY WORDS: social identity, regional identity, Antarctica, nationalism, essentialism, beliefs.

\section{INTRODUCCIÓN}

La Antártica o Antártida es conocida como el continente blanco debido a que su superficie está cubierta casi por completo de hielo. Los territorios que comprende están ubicados al sur del paralelo $60^{\circ} \mathrm{S}$ y se le considera un continente. Históricamente varios países han reclamado territorio, sin embargo luego del tratado antártico que fue firmado el 1 de Diciembre de 1959 (y que entró en vigor el 23 de junio de 1961), que regula las relaciones internacionales, las actividades hoy se centran en la investigación científica de diversas disciplinas y se le considera un territorio sin nación. Para ser exactos, son siete los países que reclaman posesión de parte del territorio antártico: Reino Unido, Nueva Zelanda, Francia, Noruega, Australia, Chile, Argentina. A partir de esto cabe cuestionarnos: ¿En qué grado para estos países la Antártica es importante en un sentido económico, territorial o identitario?

La identidad social ha sido tradicionalmente entendida, en la psicología social como una parte de lo que se es, que se deriva de pertenecer a grupos y categorías sociales. Para que esta pertenencia sea parte de la identidad, es necesario que la persona se reconozca como parte del grupo y, además, sienta conexión afectiva con él, en un proceso tradicionalmente denominado identificación grupal (Tajfel, 1984). Algunos autores han señalado que el concepto de identificación fue escrito originalmente con imprecisión lo que resultó en una falta de consenso de parte de posteriores intentos de estructurar el análisis de esta variable encontrándose en la literatura varios modelos de identificación social compuestos por distintos elementos (Scandroglio, López \& San José, 2008).

La forma en que cada individuo define su propia identidad social es un proceso complejo que incluye tanto la asimilación como la diferenciación de categorías sociales. Es el proceso de autocategorización el que determina la pertenencia psicológica a un grupo u otro, dentro de un enorme, aunque no infinito, rango de posibilidades (Ashmore, Jussim y Wilder, 2001). Dentro de las múltiples categorías que es posible considerar dentro de la identidad social tenemos aquellas referidas a la pertenencia a una región geográfica o sociopolítica. Estas identidades son creadas y mantenidas en el proceso de socialización (Arévalo, 1998). Se ha definido a la identidad regional como la parte de la identidad social que tiene relación con la historia, la cultura y el paisaje que es compartida por los miembros de una región (Lisón, 1997; Zúñiga y Asún, 2013). 
En Latinoamérica se ha puesto el énfasis en investigaciones que abordan la construcción de identidades locales y regionales a partir de un espacio real o imaginariamente delimitado, y los elementos representativos de esa identidad relacionados con el entorno físico: la naturaleza, los recursos y el clima (Buska, 2009). Algunos investigadores han postulado que los tres elementos contenidos en la definición de la identidad regional no tienen el mismo peso. Dupoivier y Schajer (1994) postulan, que la identidad regional puede establecerse aún en ausencia de una homogeneidad histórica o cultural. Estudios recientes, desarrollados en la Patagonia Austral apoyan esta idea ya que muestran, consistentemente, que el aspecto ambiental ecológico (identidad regional ecológica), presenta una mayor relevancia en el establecimiento de dicha identidad regional que los otros elementos (Santana, Carrasco y Estrada, 2013; Molina, 2011; Zuñiga y Asún, 2013).

Investigaciones han encontrado que la identidad ambiental predice las actitudes hacia el medio ambiente y el comportamiento pro-ambiental (Clayton \& Kiling, 2003). Barría y Estrada (s/f) estudiando población magallánica, encontraron que la identidad regional ecológica se conecta con una alta preocupación ambiental pero que no se traduce en comportamientos en la dirección de cuidar y proteger el medio ambiente, concluyendo que es una relación más simbólica que real. En su análisis encontraron que otras variables sociales identitarias como las creencias esencialistas y el regionalismo resultaron más relevantes que variables pro-ambientales para explicar el fenómeno de la identidad regional ambiental. Consideradas como variables constitutivas de la identidad social, el esencialismo psicológico puede ser definido como la tendencia de los sujetos a atribuir a grupos de objetos una esencia común, esencia que los determinaría (Medin, 1989). Al tratarse de grupos humanos, creer en la existencia de una esencia determinaría la presencia de una identidad social (pertenencia al grupo) que es inmutable y que puede ser comprendida como dotada de características naturales (Rothbart \& Taylor, 1992). Yzerbyt et al. (Estrada et al. 2004; Yzerbyt et al. 2001; Yzerbyt et al. 1997; Yzerbyt \& Rogier, 2001) consideran al esencialismo como una característica que podemos atribuir tanto a grupos sociales de diversos orígenes, objetivos como a creencias individuales, que además puede ser medida con cuestionarios como una variable natural y que también puede ser manipulada experimentalmente. Como fenómeno ha sido vinculado a numerosas consecuencias negativas en el plano intergrupal tales como el aumento de la visión estereotipada del otro, a percepción de diferencias difíciles de conciliar entre los grupos o incluso la infrahumanización (Martin \& Parker, 1995, Hollander \& Howard, 2000, Leyens et al. 2000; Leyens et al. 2001; Vaes, Paladino, \& Leyens, 2002). Aunque el esencialismo ha sido poco estudiado en el marco de las relaciones intragrupo ya existen una serie de investigaciones que muestran su importancia como fenómeno explicativo de fenómenos en este ámbito, tales como la estabilización de la identidad social. Según Morton y Postmes (2009) el esencialismo sería una estrategia al servicio de la identidad social ya que permitiría potenciar la oposición o apoyar otras formas de expresión de la misma (creatividad social) y le otorgaría estabilidad y cohesión. 
Tabla 1.Porcentaje de acuerdo con las afirmaciones de la escala IRA.

\begin{tabular}{|c|c|c|c|c|}
\hline & Afirmaciones & $\begin{array}{l}\text { Porcentaje } \\
\text { desacuerdo }\end{array}$ & $\begin{array}{c}\text { Porcentaje } \\
\text { neutral }\end{array}$ & $\begin{array}{c}\text { Porcentaje } \\
\text { acuerdo }\end{array}$ \\
\hline 1 & $\begin{array}{l}\text { Si la Antártica dejara de ser } \\
\text { considerada chilena, sentiria que } \\
\text { perdimos algo importante. }\end{array}$ & 3,3 & 8,0 & 88,7 \\
\hline 2 & $\begin{array}{l}\text { Pienso que la Antártica es un lugar } \\
\text { muy importante y estratégico para } \\
\text { nuestro pais, y en especial, para } \\
\text { nuestra región. }\end{array}$ & 1,7 & 15,1 & 83,2 \\
\hline 3 & $\begin{array}{l}\text { Aunque a veces me resulta dificil } \\
\text { enfrentar al clima y las condiciones } \\
\text { naturales la región, siento que este es } \\
\text { el lugar al que pertenezco. }\end{array}$ & 4,0 & 13,3 & 82,7 \\
\hline 4 & $\begin{array}{l}\text { Me siento orgulloso del entorno de la } \\
\text { Antártica, de sus paisajes y su } \\
\text { naturaleza. }\end{array}$ & 1,0 & 20,1 & 78,9 \\
\hline 5 & $\begin{array}{l}\text { Alguien que nace en la Antártica } \\
\text { también es Magallánico. }\end{array}$ & 7,0 & 17,4 & 75,5 \\
\hline 6 & $\begin{array}{l}\text { Pienso que como magallánico estoy } \\
\text { mejor preparado que otras personas } \\
\text { para resistir el clima de la Antártica. }\end{array}$ & 7,7 & 17,0 & 75,3 \\
\hline 7 & $\begin{array}{l}\text { Me siento comprometido a cuidar del } \\
\text { ambiente natural de la Antártica si } \\
\text { pudiera, porque creo que es algo que } \\
\text { me pertenece de alguna manera. }\end{array}$ & 5,4 & 19,8 & 74,8 \\
\hline 8 & $\begin{array}{l}\text { El ambiente natural de la Antártica es } \\
\text { una herencia que hemos recibido y } \\
\text { que vamos a legar a los magallánicos } \\
\text { del futuro. }\end{array}$ & 3,7 & 21,8 & 74,5 \\
\hline 9 & $\begin{array}{l}\text { Para los magallánicos seria fácil } \\
\text { adaptarse a las largas noches de } \\
\text { invierno y dias del verano en la } \\
\text { Antártica. }\end{array}$ & 9,4 & 17,7 & 72,9 \\
\hline 10 & $\begin{array}{l}\text { Me siento orgulloso/a de que la } \\
\text { Antártica y Magallanes son una } \\
\text { misma región. }\end{array}$ & 4,0 & 24,2 & 71,7 \\
\hline
\end{tabular}


11 Los magallánicos estamos en alguna medida orgullosos de la naturaleza Antártica e incluso de su clima.

$5,0 \quad 26,7 \quad 68,3$

$11,7 \quad 23,8 \quad 64,4$ también incluyen los paisajes y el clima antártico.

13 De todo aquello que significa ser magallánico, el ambiente natural de la Antártica con su clima y paisaje, es de los elementos importantes.

14 El entorno natural de la Antártica es un lugar en el que podemos realizar muchas actividades que son importantes para nosotros como región.

15 Los magallánicos compartimos la fascinación por los paisajes de la Antártica.

16 No se puede ser un verdadero magallánico sin sentirse identificado con lo antártico.

17 Sin duda, para mi, la Antártica posee los paisajes más hermosos del pais.

18 Si en conversaciones con familiares, amigos o conocidos alguien dijera algo desagradable acerca de la Antártica, yo me molestaria.

19 Me siento fuertemente ligado a la naturaleza de la Antártica.

20 Conocer el entorno natural de la Antártica marca la identidad de una persona. $\begin{array}{lll}10,4 & 31,8 & 57,9\end{array}$

$11,7 \quad 31,9 \quad 56,4$

$\begin{array}{lll}19,5 & 26,5 & 54,0\end{array}$

$\begin{array}{lll}9,0 & 40,8 & 50,2\end{array}$

$\begin{array}{lll}18,0 & 34,3 & 47,7\end{array}$

$16,7 \quad 38,3 \quad 45,0$

$21,1 \quad 35,8 \quad 43,1$

Estudios realizados en población magallánica dan cuenta de que la identidad magallánica parece estar fundada en creencias de tipo esencialista. Estos resultados han sido explicados en la dirección de que las creencias esencialistas resultan funcionales a una identidad compuesta principalmente por personas con ancestros inmigrantes y con una historia común reciente (Estrada \& Avendaño, 2008). 
El regionalismo, por su parte, puede ser entendido como la inclinación a idealizar el propio grupo, la tendencia a ser solidario con éste y a desarrollar un vínculo afectivo exacerbado, siendo valorado como superior a otros e identificándose con sus valores de forma acrítica (Roccato, Re \& Sclauzero, 2002). Como una forma específica de etnocentrismo, el regionalismo parece ser el producto de la fragmentación de la sociedad provocada por el desarrollo tecnológico que ha vuelto las fronteras de los grupos y culturas menos estables. Como consecuencia, las comunidades han tendido a desarrollar identidades más “domésticas” (Ashmore, Jussim \& Wilder, 2001). La población magallánica ha mostrado consistentemente niveles altos de regionalismo y un vínculo positivo y significativo entre esta creencia y la identidad regional ecológica (Santana, Carrasco \& Estrada, 2013). Dentro de las interpretaciones dadas a este fenómeno se encuentra la característica particular del territorio de este grupo social que involucra un aislamiento geográfico relativo, que facilita la identificación con la Región.

Investigaciones que abordan los factores que influirían en el desarrollo de actitudes favorables hacia la naturaleza, postulan que el acceso a la misma sería explicativa de estas actitudes, exclusivamente en la medida en que se relacionan con un contacto afectivamente gratificante (Roczen, Duvier, Bogner \& Kaiser, 2012). De acuerdo a lo planteado por Holahan (2008), que adhiere a un Modelo Unidimensional afectivo del estudio de la actitud, las actitudes ambientales serían originadas por los sentimientos favorables o desfavorables que se poseen hacia algunas características del ambiente físico o hacia un problema relacionado con él.

Sin embargo, no existen estudios sobre la construcción de identidades territoriales o regionales que consideren espacios con los que no se tiene un contacto directo o real, como podría ser el caso de la Antártica que es visitada por un escaso número de personas. Entonces, ¿En qué medida las experiencias gratificantes o directas, el conocimiento real o simbólico serían elementos necesarios para el desarrollo de una actitud positiva e identificación con la Antártica?, es la pregunta que guía la presente investigación. De manera que el objetivo se centra en profundizar respecto a la conformación identitaria asociada a un espacio geográfico con el que no se tiene contacto ni experiencia directa, y la relación de esta identidad con el conocimiento que se tiene de dicho territorio, así como con variables sociales ya vinculadas con este tipo de identidad regional. En términos más simples, la hipótesis que guía este estudio es que un grupo de personas puede construir una identidad social regional a partir de un territorio con el que no se tiene contacto real.

\section{MÉTODO}

Participantes: La muestra, no probabilística accidental, estuvo compuesta de 300 participantes, que se auto categorizaron como magallánicos, todos ellos mayores de edad, en un rango entre 18 a 81 años, $(M=34,4$ y la $D T=15,5)$, residentes en la región de Magallanes. El $69,7 \%$ fue de género femenino mientras que el 30,3\% restante pertenece al género masculino. 
Diseño: No experimental, transversal correlacional.

Instrumentos: Escala de Identidad Regional Antártica (ver Tabla 1). Es una adaptación hecha de la Escala de Identidad Regional Ecológica (Santana, Carrasco y Estrada, 2013). Se constituye de un total de 20 afirmaciones que miden la identificación con el entorno ambiental antártico que se responden sobre una escala Likert (1: Totalmente en desacuerdo hasta 5: Totalmente de acuerdo. Para esta muestra alcanzó un 0,92 de fiabilidad (Alfa de Cronbach).

Cuestionario de conocimientos básicos sobre la Antártica (Estrada, 2013) que está compuesto por 12 preguntas de dificultad diversa, elaboradas para este estudio y que miden el conocimiento respecto a cuestiones de orden geográfico y sociopolítico de la Antártica (ver Tabla 3). El instrumento fue validado por pares expertos (psicólogos investigadores en psicología social) y su confiabilidad determinada luego de una aplicación piloto.

Escala de Regionalismo: Adaptación del instrumento de Nacionalismo de Roccato, Re y Sclauzero (2002). Compuesto originalmente de seis afirmaciones que miden el nacionalismo y que durante la adaptación se aumentaron a ocho afirmaciones con el objetivo de medir la categoría de regionalismo magallánico. Los ítems se responden en base a una escala Likert (1: Totalmente en desacuerdo hasta 5: Totalmente de acuerdo). Presentó una confiabilidad de 0,86 (Alfa de Cronbach).

Escala de Esencialismo Psicológico (Estrada, 2003). La escala está compuesta por 11 afirmaciones que se basan en la descripción que hace Yzerbyt y Schadron (1996) del síndrome esencialista (Calquin \& Estrada, 2011). Estas fueron evaluadas a través de una escala de tipo Likert de cinco puntos donde 1 significaba para nada de acuerdo y 5 totalmente de acuerdo. La consistencia interna fue de 0,94 (Alfa de Cronbach) para esta muestra.

Procedimiento: Los participantes fueron contactados en sus lugares de estudio y trabajo. Estos aceptaron voluntariamente a participar en el estudio contestando los 4 instrumentos simultáneamente de forma individual y autoaplicada. Firmaron un consentimiento informado y al finalizar la aplicación se contestó a sus preguntas y se les agradeció por su participación.

\section{RESULTADOS}

La medición de la Identidad Regional Antártica, mostró un promedio de 3,84 (DE: 0,59) con un mínimo observado de 2,50 a un máximo de 5, 00. Dicho promedio resultó levemente inferior al reportado en investigaciones recientes en que se midió la Identidad Regional Magallánica, $M=4, t_{(299)}=-4,37, p=0,0001$ (Santana, Carrasco y Estrada, 2013). Las afirmaciones que tuvieron mayor porcentaje de acuerdo fueron: "Si la Antártica dejara de ser considerada Chilena, sentiría que perdemos algo importante”, con un porcentaje de 88,7\% de acuerdo; "Pienso que la Antártica es un lugar muy importante y estratégico para 
nuestro país y en especial para nuestra región” con 83,2\%; “Aunque a veces me resulta difícil enfrentar al clima y las condiciones naturales de mi región, siento que este es el lugar al que pertenezco" con $82,7 \%$. Las que tuvieron un mayor porcentaje de desacuerdo fueron: "Conocer el entorno natural de la Antártica marca la identidad de una persona" con un 21,1\% de desacuerdo; "No se puede ser un verdadero magallánico sin sentirse identificado con lo antártico" con un 19,5\%; "Si en conversaciones con familiares, amigos o conocidos alguien dijera algo desagradable sobre la Antártica, yo me molestaría” con un $18,0 \%$.

Respecto al conocimiento sobre la Antártica, se observó un promedio de respuestas correctas de 5,40 de un máximo posible de 12 (DE: 2,22) con un rango observado de 0 a 12. Entre las afirmaciones que resultaron más fáciles de contestar se encuentran: "La Universidad de Magallanes hace estudios sobre la Antártica” donde el 82,1\% de sujetos respondió afirmativamente, "Existe algún acuerdo internacional para proteger y cuidar la Antártica”, con 68,4\%; y "Su superficie está casi completamente cubierta de hielo y nieve” con $66,8 \%$. Por otra parte, las 3 afirmaciones que resultaron más difíciles fueron: "Parte de la Antártica le pertenece legalmente a Chile”, donde el 91,5\% de las personas respondió de manera incorrecta, "Chile tiene más de 8 bases en la Antártica”, con 84,4\% y "La Antártica es más grande que Europa”; con 85,3\%.

La Escala de Regionalismo mostró un promedio de 4,08 (DE: 0,69) con un mínimo observado de 2,09 a un máximo de 5,00. Comparada con un estudio anterior la muestra resultó similar a lo observado anteriormente, $M=4,03 t_{(299)}=1,25, p=0,21$, n.s. En otros términos, el promedio de regionalismo observado es típico para la población.

El esencialismo psicológico alcanzó un promedio de 4,05 (DE: 0,71) con un mínimo de 2,09 a un máximo observado de 5,00. Dicho promedio resultó similar reportado en investigaciones recientes (Estrada y Avendaño, 2008), $M=3,99 t_{(353)}=2,15, p=0,03$.

Las correlaciones entre las variables del estudio muestran que la Identidad Regional Antártica (IRA) se asocia positivamente tanto con el regionalismo como con el esencialismo y el nivel de conocimiento (ver tabla 2). Mientras que la IRA muestra niveles equivalentes de asociación tanto con el regionalismo como con el esencialismo ( $Z=-0,98, p=0,33$, n.s.), la relación de esta variable con el nivel de conocimiento resulta significativamente menor.

Al regresar a la variable Identidad Regional Antártica según las tres variables principales del estudio, esto es, esencialismo, regionalismo y nivel de conocimiento, todas ellas resultan significativas explicando un $44 \%$ de la varianza, $F_{(3,294)}=78,01, p=$ 0,0001 . Las creencias esencialistas $(\beta=0,45, \mathrm{p}=0,0001,95 \%$ IC $[0,26,0,49])$ resultan más explicativas que el regionalismo $(\beta=0,24, \mathrm{p}=0,0001,95 \%$ IC $[0,09,0,32])$ y éste a su vez que el nivel de conocimiento $(\beta=0,10, \mathrm{p}=0,02,95 \%$ IC $[0,09,0,05])$. En otros términos, el nivel de conocimiento sobre lo antártico es explicativo de la identidad regional antártica, pero es la menos importante de las tres variables. 
ESTUDIO SOBRE LA IDENTIDAD MAGALLÁNICA ANTÁRTICA

Tabla 2. Correlaciones entre las variables del estudio.

\begin{tabular}{|c|c|c|c|c|c|}
\hline & Variables & 1 & 2 & 3 & 4 \\
\hline 1 & Identidad Regional Antártica & - & $* * 0,59$ & $* * 0,64$ & $* * 0,15$ \\
\hline 2 & Regionalismo & & - & $* * 0,77$ & 0,10 \\
\hline 3 & Esencialismo & & & - & 0,07 \\
\hline 4 & Conocimientos & & & & - \\
\hline
\end{tabular}

* Significativa al $0,05,{ }^{* *}$ significativa al 0,01

Fuente: Elaboración propia.

Tabla 3. Porcentajes de respuestas correctas e incorrectas en conocimientos básicos sobre la Antártica.

\begin{tabular}{|c|c|c|c|}
\hline & Afirmaciones & $\begin{array}{l}\text { Porcentaje } \\
\text { incorrecto }\end{array}$ & $\begin{array}{l}\text { Porcentaje } \\
\text { Correcto }\end{array}$ \\
\hline 1 & $\begin{array}{l}\text { La Universidad de Magallanes hace estudios sobre } \\
\text { la Antártica. }\end{array}$ & 17,9 & 82,1 \\
\hline 2 & $\begin{array}{l}\text { Existe algún acuerdo internacional para proteger y } \\
\text { cuidar la Antártica. }\end{array}$ & 31,6 & 68,4 \\
\hline 3 & $\begin{array}{l}\text { Su superficie está casi completamente cubierta de } \\
\text { hielo y nieve. }\end{array}$ & 33,2 & 66,8 \\
\hline 4 & $\begin{array}{l}\text { En las bases participan la INACH, el ejército, la } \\
\text { armada y la FACH. }\end{array}$ & 33,9 & 66,1 \\
\hline 5 & Antártica y Antártida se refieren al mismo lugar. & 37,3 & 62,7 \\
\hline 6 & Hay osos polares en la Antártica. & 39,7 & 60,3 \\
\hline 7 & Comúnmente llueve en la Antártica. & 62,8 & 37,2 \\
\hline 8 & Cualquier persona puede viajar a la Antártica. & 67,8 & 32,2 \\
\hline 9 & $\begin{array}{l}\text { Hay tierras, ríos, lagos y montañas debajo del hielo } \\
\text { de la Antártica. }\end{array}$ & 67,9 & 32,1 \\
\hline 10 & Chile tiene más de 8 bases en la Antártica. & 84,4 & 15,6 \\
\hline 11 & La Antártica es más grande que Europa. & 85,3 & 14,7 \\
\hline 12 & $\begin{array}{l}\text { Parte de la Antártica le pertenece legalmente a } \\
\text { Chile. }\end{array}$ & 91,5 & 8,5 \\
\hline
\end{tabular}

Fuente: Elaboración propia. 


\section{DISCUSIÓN}

Nuestro estudio tuvo como objetivo investigar si una población como la magallánica puede poseer una identidad asociada a un territorio en ausencia de experiencias directas e incluso de información general sobre éste, como podría ser el caso del territorio antártico. La hipótesis que lo guió fue que un grupo de personas puede construir su identidad social regional incluyendo a un territorio con el que no se tiene contacto ni del que se posee un conocimiento real.

Los resultados principales indican que los magallánicos poseen una alta identificación con el territorio Antártico. Tal y como lo plantea Holahan (2008), la identificación es un proceso afectivo, por lo tanto, no requiere necesariamente de la presencia de elementos racionales. En este sentido, "sentirse parte" o "sentir algo parte de lo que se es" puede construirse de una forma simbólica que no se ancla en una experiencia directa o concreta. Esta idea se ve reafirmada por la relación observada entre la identidad regional Antártica y el conocimiento respecto a este territorio entendido como un espacio geopolítico, porque, aunque existente, este vínculo mostró ser bajo. Sólo en un poco más del 2\% de los casos, la identidad social magallánico-antártica se asocia al conocimiento sobre características generales de este territorio, su clima y la organización de las tareas que allí se realizan. La existencia de una identidad Antártica en la población Magallánica contradice la hipótesis de que la actitud positiva hacia cierto territorio estaría asociada a las experiencias gratificantes que se hayan tenido en relación a éste (Roczen, Duvier, Bogner, y Kaiser, 2012).

Por otra parte, al intentar dar cuenta de este aspecto de la identidad social, surgen como principales variables explicativas aquellas que hemos denominado las variables sociales identitarias, estas son, el esencialismo y el regionalismo. Tal como fue observado en un estudio anterior (Barría y Estrada, en prensa) las creencias esencialistas y regionalistas poseen un mayor peso explicativo del vínculo identitario asociado al entorno natural. En dicho estudio, variables proambientales mostraron ser menos relevantes que las socioidentitarias. Incluso, aquellas variables proecológicas asociadas positivamente lo hicieron desde los aspectos que evaluaron sentimientos favorables hacia el entorno natural. Buscando respuesta al peso de estas variables una explicación posible es que en el aprendizaje escolar, los medios de comunicación y la cultura en general el nombre formal de la región es "Región de Magallanes y de la Antártica Chilena”, esto combinado con las creencias esencialistas provoca que ambos territorios se perciban como uno solo, que comparte una misma esencia. En cuanto al regionalismo uno de sus componentes es el desarrollo de un vínculo afectivo exacerbado con el grupo lo que se corresponde con el modelo de Holahan (2008) mencionado anteriormente. Por otra parte, si consideramos que el esencialismo y el regionalismo son variables constituidas por ideas no necesariamente racionales, asociadas a la identidad social de las personas, resulta coherente pensar que, la presencia de estas creencias en la población magallánica puede dar cuenta de la baja importancia que el conocimiento real y concreto tiene en la formación de la identidad antártica. 
En otros términos, los resultados del presente estudio parecen dar apoyo empírico a la hipótesis de que parte de la identidad social regional no se construye a partir de una conexión con entorno natural sino que con creencias simbólicas respecto a lo que esto representa para los habitantes de un territorio. En este sentido, no es posible aún establecer si este fenómeno es particular a la población estudiada o es generalizable a otras identidades regionales. Una primera limitación del presente estudio, entonces, es el uso de una muestra homogénea que consideró como elementos constituyentes a personas de una única identidad regional que presenta, probablemente por sus características geográficas y climatológicas (Patagonia Austral), un perfil diferencial que no necesariamente será observado en otras poblaciones. Futuras investigaciones deberán ocuparse de esta situación de forma de realizar estudios trans-regionales incluyendo nuevas variables tanto tradicionalmente vinculadas con el entorno físico como con la identidad social. Una segunda etapa contempla ampliar la muestra incorporando a otros sectores de la población magallánica, además de incorporar al estudio a participantes que han estado en el territorio antártico, para así observar el impacto posible que el contacto directo pudiese tener en la formación de la identidad social regional - antártica.

\section{REFERENCIAS}

Arévalo, J. (1998). La identidad extremeña. Reflexiones desde la antropología social. Gazeta de Antropología, 14, 04.

Ashmore, R. D., Jussim L., \& Wilder, D. (2001). Social identity, intergroup conflict, and conflict reduction. New York: Oxford University Press.

Barría, M. J., \& Estrada, C. (s/f) Identidad Regional Ecológica: ¿Más identidad regional que ecológica?. Escrito no publicado. Centro de Estudios en Psicología, Departamento de Psicología, Universidad de Magallanes.

Buska, S. (2009). Identidades regionales y locales. Disponible en: http://historia.ihnca.edu. ni/ccss/dmdocuments/conferencias/CCSS2009/Ponencia_Soili_Buska_Identidades_ regionales_y_locales.pdf

Calquin, D., \& Estrada, C. (2011). Esencialismo, su Impacto en la Percepción de Similitud y el Uso de Diferente Tipo de Información en la Formación de Impresión. Psykhe, 20(1), 63-75.

Clayton, S., \& Kiling, A. (2003). La preocupación por el medio ambiente y el comportamiento pro-ambiental en Turquía: el papel de la identidad nacional y ambiental. Psyecology, 4(3), 311-330.

Dupovier, E., \& Schajer, H. (1994). La identidad regional: Problemas teóricos. Perspectivas políticas. L'identité politique (445 pp.). Presses Universitaires de France, Paris.

Estrada, C. (2003). Limpact de l'essentialisme psychologique sur les processus intra- et intergroupes [El impacto del esencialismo psicológico en los procesos intra e 
intergrupales] (Tesis de Doctorado no publicada), Faculté de Psychologie et des Sciences de l' Education, Université catholique de Louvain, Bélgica.

Estrada, C., \& Avendaño, S. (2008). ¿Consideran los magallánicos que su cultura posee una esencia única?: Estudio descriptivo de la aplicación de un instrumento de evaluación de esencialismo cultural a una muestra puntarenense. Magallania, 36(2), 155-162.

Estrada, C., Yzerbyt, V. \& Seron, E. (2004). El efecto del esencialismo psicológico sobre las teorías ingenuas de las diferencias grupales. Psicothema, 16(2), 181-186.

Holahan, C. (2008). Psicología Ambiental un Enfoque General. México: Limusa.

Hollander, J. A., \& Howard, J. A. (2000). Social psychological theories on social inequalities. Social Psychology Quarterly, 63, 338-351.

Leyens, J. P., Paladino, P. M., Rodriguez-Torrez, R., Vaes, J., Demoulin, S., Rodriguez-Perez, A., \& Gaunt, R. (2000). The emotional side of prejudice: The attribution of secondary emotions to ingroups and outgroups. Personality and Social Psychology Review, 4, 186197.

Leyens, J. P., Paladino, P. M., Rodriguez-Torrez, R., Vaes, J., Demoulin, S., Rodriguez-Perez, A., \& Gaunt, R. (2000). The emotional side of prejudice: The attribution of secondary emotions to ingroups and outgroups. Personality and Social Psychology Review, 4(2), 186-197.

Lisón, C. (1997). Las máscaras de la identidad. Claves antropológicas. Barcelona: Ariel.

Martin, C. L., \& Parker, S. (1995). Folk theories about sex and race differences. Personality and Social Psychology Bulletin, 21(1), 45-57.

Medin, D. L. (1989). Concepts and conceptual structure. American Psychologist, 44(12), 14691481.

Molina, W. (2011). Identidad Regional en Magallanes, sus expresiones simbólicas y territoriales. Magallania, 39(1), 59-69.

Morton, T. A., \& Postmes, T. (2009). When differences become essential: Minority essentialism in response to majority treatment. Personality and Social Psychology Bulletin, 35(5), 656-668.

Roccato, M., Re, M., \& Sclauzero, S. (2002). Autoritarismo, Nacionalismo y Patriotismo. Un estudio con militantes italianos. Psicología Política, 25(1), 21-35.

Roczen, N., Duvier, C., Bogner, F., \& Kaiser, F. (2012). La búsqueda de los posibles orígenes de una actitud favorable hacia la naturaleza. Psyecology, 3(3), 287-298.

Rothbart, M. \& Taylor, M. (1992). Category labels and social reality: Do we view social categories as natural kinds? En K. Semin y A. Fiedler (Eds.). Language, interaction and social cognition (pp.11-36). London: Sage.

Santana, D., Carrasco, H., \& Estrada, C. (2013). La identidad ecológica: el rol del entorno ambiental en la construcción de la identidad patagónica. Estudios de Psicología, 34(1), 83- 88.

Scandroglio, B., López, J., \& San José, M. (2008). La Teoría de la Identidad Social: una síntesis 
crítica de sus fundamentos, evidencias y controversias. Psicothema, 20(1), 80-89. Tajfel, H. (1984). Grupos humanos y categorías sociales. Barcelona: Editorial Herder S.A. Vaes, J., Paladino, P. M., \& Leyens, J. P. (2002). The lost e-mail: Prosocial reactions induced by uniquely human emotions. British Journal of Social Psychology, 41(4), 521-534.

Yzerbyt, V., Corneille, O., \& Estrada, C. (2001). The interplay of subjective essentialism and entitativity in the formation of stereotypes. Personality and Social Psychology Review, 5(2), 141-155.

Yzerbyt, V., Rocher, S., \& Schadron, G. (1997). Stereotypes as explanations: A subjective essentialistic view of group perception. En R. Spears, P. Oakes, N. Ellemers y S. A. Haslam (Eds.), The social psychology of stereotyping and group life (pp. 20-50). Oxford: Blackwell published.

Yzerbyt, V., \& Rogier, A. (2001). Blame it on the group: Entitativity, subjective essentialism and social attribution. En J. Jost \& B. major (Eds.), The psychology of legitimacy: Emerging perspectives on ideology, justice and intergroup relations (pp. 20-49). New York: Cambridge University Press.

Yzerbyt, V., \& Schadron, G. (1996). Connaitre et juger autrui: Une introduction à la cognition sociale [Conocer y juzgar al otro: una introducción a la cognición social]. Grenoble, Francia: Presses Universitaires de Grenoble.

Zúñiga, C., \& Asún, R. (2013). Identidad regional en estudiantes universitarios de Magallanes: Una aproximación psicosocial. Magallania. 41(1), 3-98. 
